\title{
Hydrogen Burning in Low Mass Stars Constrains Scalar-Tensor Theories of Gravity
}

\author{
Jeremy Sakstein" \\ Institute of Cosmology and Gravitation, University of Portsmouth, Portsmouth PO1 3FX, United Kingdom
}

(Received 26 June 2015; published 10 November 2015)

\begin{abstract}
The most general scalar-tensor theories of gravity predict a weakening of the gravitational force inside astrophysical bodies. There is a minimum mass for hydrogen burning in stars that is set by the interplay of plasma physics and the theory of gravity. We calculate this for alternative theories of gravity and find that it is always significantly larger than the general relativity prediction. The observation of several low mass red dwarf stars therefore rules out a large class of scalar-tensor gravity theories and places strong constraints on the cosmological parameters appearing in the effective field theory of dark energy.
\end{abstract}

DOI: 10.1103/PhysRevLett.115.201101

PACS numbers: 04.50.Kd, 26.20.Cd, 95.36.+x, 98.80.-k

The dark energy and cosmological constant problems have been driving the study of alternative theories of gravity as a possible explanation for the acceleration of the cosmic expansion (see Ref. [1] for a recent review). Among them, scalar-tensor theories are particularly well studied, which raises the question of what is the most general theory that is free of the Ostrogradsky ghost instability. This has recently been answered by Gleyzes, Langlois, Piazza, and Vernizzi (GLPV) [2]. The GLPV framework encapsulates every healthy scalar-tensor theory, and a large subclass, the beyond Horndeski class, admits self-accelerating solutions that are viable competitors to $\Lambda \mathrm{CDM}[3,4]$. These theories have a peculiar property: they make identical predictions to general relativity (GR) except that they predict a weakening of the strength of gravity inside astrophysical bodies [5]. This has an important implication which has hitherto been left unexplored: the minimum mass for hydrogen burning (MMHB) is larger than the observed mass of several hydrogen burning red dwarf stars. Low mass stellar objects do not achieve the necessary core conditions to ignite hydrogen, but heavier objects are hotter and denser. Stars heavier than the MMHB fuse hydrogen and are classified as red dwarfs; those with lower masses are classified as brown dwarfs. General relativity predicts that the MMHB is $0.08 M_{\odot}[6,7]$, and, indeed, several red dwarfs have been observed with masses larger than $0.08 M_{\odot}$ (see Ref. [8] and references therein). In this Letter, we calculate the MMHB predicted by the most general scalar-tensor theories. The reduced strength of gravity has the effect that hydrostatic equilibrium can be maintained at lower core temperatures and densities. As a consequence, we find that the MMHB can be significantly larger than observed in nature and therefore a large class of these models can be ruled out. A large majority of current dark energy research focuses on models that are subsets of the GLPV theory [9]. Therefore, our results have important implications for scalar-tensor dark energy models. This is the first time that the observation of hydrogen burning in stars has been used to constrain the theory of gravity.
The structure of low mass stellar objects makes them perfect probes of modified gravity. They have uniform compositions and have properties that are only weakly dependent on nongravitational physics, such as variations in their metallicity and opacity. This means they are free of the degeneracies that plague some, $[10,11]$ but not all [12], stellar structure tests of gravity. Furthermore, their equation of state is well known and is relatively simple. This means that the polytropic techniques developed by Refs. [13,14] can be applied to calculate the MMHB analytically. References $[13,14]$ have shown that scalar-tensor theories predict that the hydrostatic equilibrium equation is modified to

$$
\frac{d P}{d r}=-\frac{G M \rho}{r^{2}}-\frac{\Upsilon}{4} G \rho \frac{d^{2} M}{d r^{2}} .
$$

$\Upsilon$ is the only free dimensionless parameter that characterizes the deviations from GR. When $\Upsilon>0$, the new term is negative because the mass is more concentrated in the center of the star; therefore, the strength of gravity is reduced compared with GR.

The parameter $\Upsilon$ is directly related to the parameters $\alpha_{B}$, $\alpha_{T}$, and $\alpha_{H}$ appearing in the effective field theory (EFT) of dark energy [15] via [14]

$$
\Upsilon=\frac{4 \alpha_{H}^{2}}{\alpha_{H}-\alpha_{T}-\alpha_{B}\left(1+\alpha_{T}\right)} .
$$

The five parameters appearing in the EFT completely characterize the linear cosmology of the theory. (Note that there are two parameters, $\alpha_{K}$ and $M$, that are not constrained by the effects presented here.) Any independent constraint on $\Upsilon$ therefore constrains this combination of the cosmological parameters, complementing searches on other scales and restricting the possible deviations from GR. One example of a well-studied and viable alternative to GR is the covariant quartic Galileon [3], which admits selfaccelerating solutions without the need for a cosmological 
constant. This theory has $\Upsilon=1 / 3$, which, as we will see below, is ruled out by the constraint we will ultimately obtain using the MMHB.

Stars are the result of the complex interplay between many different areas of physics, but this is the only stellar structure equation where the modifications of GR appear. The rest of the physics that determines the structure and evolution of the star-energy generation from nuclear burning, the equation of state, the opacity and composition-is nongravitational and is hence unaltered by changing the theory of gravity.

Low mass stellar objects (see Ref. [7] for a review of the science of brown dwarfs and other low mass stellar objects) are supported by a combination of thermal and electron degeneracy pressure and are well described by the polytropic equation of state [7]

$$
P=K \rho^{5 / 3}, \quad K=\frac{\left(3 \pi^{2}\right)^{2 / 3} \hbar^{2}}{5 m_{e} m_{\mathrm{H}}^{5 / 3} \mu_{e}^{5 / 3}}\left(1+\frac{\alpha}{\eta}\right),
$$

where $m_{e}$ and $m_{\mathrm{H}}$ are the electron and hydrogen mass, respectively, $\mu_{e}=1.143$, and $\alpha=4.82$. $\eta$ is a measure of the degeneracy of the star. It is formally defined as the ratio of the Fermi energy to $k_{B} T$ and is given by

$$
\eta=\frac{\left(3 \pi^{2}\right)^{2 / 3} \hbar^{2}}{2 m_{e} m_{\mathrm{H}}^{2 / 3} k_{B}} \frac{\rho^{2 / 3}}{\mu_{e}^{2 / 3} T} .
$$

$\eta$ is a constant throughout the entire star. Defining the dimensionless radial coordinate and density variables

$$
r=r_{c} \xi, \quad r_{c}{ }^{2}=\frac{5 K}{8 \pi G} \rho_{c}^{2}, \quad \rho=\rho_{c} \theta^{3 / 2},
$$

where $\rho_{c}$ is the central density, the structure of the star is governed by the Lane-Emden equation (LEE) [13]

$\frac{1}{\xi^{2}} \frac{d}{d \xi}\left[\left(1+\frac{3 \Upsilon \xi^{2} \theta^{1 / 2}}{2}\right) \xi^{2} \frac{d \theta}{d \xi}+\frac{\Upsilon}{2} \xi^{3} \theta^{3 / 2}\right]=-\theta^{3 / 2}$.

The boundary conditions are $\theta(0)=1$ and $\theta^{\prime}(0)=0$, which imply that, near the origin,

$$
\theta(\xi) \approx 1-\frac{1}{6}\left(1+\frac{3 \Upsilon}{2}\right) \approx \exp \left[-\frac{1}{6}\left(1+\frac{3 \Upsilon}{2}\right)\right] .
$$

The radius of the star $R$ is defined by $\rho(R)=0$, which defines $\xi_{R}$ such that $\theta\left(\xi_{R}\right)=0$. The structure of the star is described by solutions of the LEE, which predicts the stellar radius, the mass-radius relation, and the central density:

$$
M=4 \pi r_{c}^{3} \rho_{c} \omega, \quad R=\gamma \frac{K}{G M^{1 / 3}}, \quad \rho_{c}=\delta \frac{3 M}{4 \pi R^{3}},
$$

where the $(\Upsilon$-dependent) structure coefficients are

$$
\omega \equiv-\left.\xi_{R}^{2} \frac{d \theta}{d \xi}\right|_{\xi=\xi_{R}}, \quad \gamma \equiv\left[\frac{125}{128 \pi^{2}}\right]^{\frac{1}{3}} \omega^{\frac{1}{3} \xi_{R}},
$$

and

$$
\delta \equiv-\frac{\xi_{R}}{3 d \theta /\left.d \xi\right|_{\xi=\xi_{R}}} .
$$

These quantities then encode the effects of scalar-tensor theories on the stellar properties.

The rate-limiting reaction for hydrogen burning is the weak process $p+p \rightarrow d+e^{+}+\nu_{e}$. The energy generation rate (per unit mass) at typical brown dwarf temperatures and densities can be approximated by the power law [7]

$$
\epsilon_{\mathrm{HB}}=\epsilon_{c}\left(\frac{T}{T_{c}}\right)^{s}\left(\frac{\rho}{\rho_{c}}\right)^{u-1}, \quad \epsilon_{c}=\epsilon_{0} T_{c}^{s} \rho_{c}{ }^{u-1},
$$

with $s \approx 6.31, u \approx 2.28$, and $\epsilon_{0}=3.4 \times 10^{-9} \mathrm{erg} \mathrm{g}^{-1} \mathrm{~s}^{-1}$. Our procedure for calculating the MMHB will then be the following: We will first calculate the luminosity in hydrogen burning, $L_{\mathrm{HB}}$, by integrating Eq. (11) over the entire star using solutions of the LEE. Next, the luminosity at the photosphere $L_{e}$ is calculated using the Stefan-Boltzmann law. The MMHB is the lowest mass where the reaction above can be sustained stably; i.e., energy generated in the core is compensated by energy radiated from the surface, which corresponds to the mass where $L_{\mathrm{HB}}=L_{e}$. Demanding that the two are equal then gives us a condition for the mass.

We begin with the luminosity in hydrogen burning. Since $\eta$ is a constant, we have $T / T_{c}=\left(\rho / \rho_{c}\right)^{2 / 3}$ and so, using Eqs. (5) and (11), we have

$$
L_{\mathrm{HB}}=4 \pi r_{c}^{3} \rho_{c} \epsilon_{c} \int_{0}^{\xi_{R}} \xi^{2} \theta^{3 / 2 u+s} d \xi .
$$

This can be integrated using Eqs. (7) and (8) to find

$$
L_{\mathrm{HB}}=\frac{3 \sqrt{\pi}}{\sqrt{2} \omega\left[\left(1+\frac{3 \Upsilon}{2}\right)\left(\frac{3}{2} u+s\right)\right]^{3 / 2}} \epsilon_{c} M .
$$

Using Eqs. (3) and (8), one has

$$
\rho_{c}=\frac{125 G^{3} m_{e}^{3} m_{\mathrm{H}}^{5} \mu_{e}^{5}}{12 \pi^{5} \hbar^{6}} \frac{\delta}{\gamma^{3}} M^{2}\left(1+\frac{\alpha}{\eta}\right)^{-3}
$$

and

$$
T_{c}=\frac{25 G^{2} m_{e} m_{\mathrm{H}}^{8 / 3} \mu_{e}^{8 / 3}}{2^{7 / 3} \pi^{2} k_{B} \hbar^{2}} \frac{M^{4 / 3}}{\gamma^{2}} \frac{\eta}{(\alpha+\eta)^{2}},
$$

which can be used in Eq. (11) to find $\epsilon_{c}$. The luminosity in hydrogen burning is then 
$L_{\mathrm{HB}}=5.2 \times 10^{6} L_{\odot} \frac{\delta^{5.487}}{\omega \gamma^{16.46}\left(1+\frac{3 \Upsilon}{2}\right)^{3 / 2}} M_{-1}^{11.973} \frac{\eta^{10.15}}{(\eta+\alpha)^{16.46}}$,

where $M_{-1}=M / 0.1 M_{\odot}$.

Our next task is to compute the luminosity at the photosphere. Using numerical modeling of the equation of state of hydrogen at high densities, it has been shown that the temperature and density at the photosphere are related via [7]

$$
\frac{T_{e}}{\mathrm{~K}}=\frac{1.8 \times 10^{6}}{\eta^{1.545}}\left(\frac{\rho_{e}}{\mathrm{~g} / \mathrm{cm}^{3}}\right)^{0.42} .
$$

The photosphere is very thin compared with the radius of the star, and so the surface gravity, $g=G M(r) / r^{2}$, can be treated as a constant to a high degree of accuracy. The location of the photosphere is the radius where the optical depth, defined as

$$
\tau(r)=\int_{r_{e}}^{\infty} \kappa \rho d r
$$

where $\kappa$ is the Rosseland mean opacity, is equal to $2 / 3$. In GR, this is found by substituting for the density using the hydrostatic equilibrium equation (1) and integrating using the fact that $g$ is constant. In our theory, there is the additional complication coming from the term proportional to $d^{2} M / d r^{2}$. We will deal with this using the same approximation as is made in the GR calculation, i.e., assuming that $g$ is constant. This implies that $d\left(M / r^{2}\right) / d r=0$, and, hence, we have

$$
\frac{d M}{d r}=2 \frac{M}{r} \quad \text { and } \quad \frac{d^{2} M}{d r^{2}}=2 \frac{M}{r^{2}} .
$$

The hydrostatic equilibrium in the vicinity of the photosphere is then

$$
\frac{d P_{e}}{d r}=-g \rho\left(1+\frac{\Upsilon}{4}\right)
$$

Substituting this into Eq. (18), we find

$$
P_{e}=\frac{2}{3 \kappa}\left(1+\frac{\Upsilon}{4}\right) g=\frac{\rho_{e} k_{B} T_{e}}{\mu m_{\mathrm{H}}},
$$

where the ideal gas law has been used and the mean molecular mass $\mu=0.593$ corresponding to a hydrogenhelium mixture. Using Eq. (8), the surface gravity is

$$
g=3.15 \times 10^{6} \gamma^{-2} M_{-1}^{5 / 3}\left(1+\frac{\alpha}{\eta}\right)^{-2} \mathrm{~cm} / \mathrm{s}^{2} .
$$

Using this in Eq. (21) and replacing the pressure using the polytropic relation (5), we find the density at the photosphere,

$\frac{\rho_{e}}{\mathrm{~g} / \mathrm{cm}^{3}}=5 \times 10^{-5} M_{-1}^{1.17}\left[\frac{\left(1+\frac{\Upsilon}{4}\right)}{\kappa_{-2}}\right]^{0.7} \frac{\eta^{1.09}}{\gamma^{1.41}}\left(1+\frac{\alpha}{\eta}\right)^{-1.41}$,

where $\kappa_{-2}=\kappa / 10^{-2} \mathrm{~cm}^{2} / \mathrm{g}$. This can then be used in conjunction with Eq. (17) to find the effective temperature:

$\frac{T_{e}}{\mathrm{~K}}=2.9 \times 10^{4} \frac{M_{-1}^{0.49}}{\gamma^{0.59} \eta^{1.09}}\left[\frac{\left(1+\frac{\Upsilon}{4}\right)}{\kappa_{-2}}\right]^{0.296}\left(1+\frac{\alpha}{\eta}\right)^{-0.59}$.

Using these in the formula $L_{e}=4 \pi R^{2} \sigma T_{e}^{4}$, we find

$L_{e}=2.65 L_{\odot} \frac{M_{-1}^{1.305}}{\gamma^{2.366} \eta^{4.351}}\left[\frac{\left(1+\frac{\Upsilon}{4}\right)}{\kappa_{-2}}\right]^{1.183}\left(1+\frac{\alpha}{\eta}\right)^{-0.366}$.

The condition for stable hydrogen burning is $L_{\mathrm{HB}}=L_{e}$ and so, using Eqs. (16) and (25). we have

$3.76 M_{-1}=\left[\frac{\left(1+\frac{\Upsilon}{4}\right)}{\kappa_{-2}}\right]^{0.11}\left(1+\frac{3 \Upsilon}{2}\right)^{0.14} \frac{\gamma^{1.32} \omega^{0.09}}{\delta^{0.51}} I(\eta)$,

with

$$
I(\eta) \equiv \frac{(\alpha+\eta)^{1.509}}{\eta^{1.325}}
$$

From here on, we set $\kappa_{-2}=1$, which is typical for high mass brown dwarfs. The stellar composition does not vary between different stars by large amounts, and, since Eq. (26) is only a weak function of the opacity, deviations from this value are highly subdominant to modified gravity effects. Importantly, the function $I(\eta)$ has a unique minimum value of 2.34 when $\eta=34.7$. This means that if $M_{-1}$ is too low, there is no consistent solution to Eq. (26); the MMHB is the smallest mass for which Eq. (26) is satisfied. In GR, one has $\gamma=2.357, \delta=5.991$, and $\omega=2.714$, which gives $M_{\mathrm{MMHB}}^{\mathrm{GR}} \approx 0.0845 M_{\odot}$. This is extremely close to the results of detailed numerical simulations [6], which predict a value of $0.075 M_{\odot}$. In Fig. 1 we plot the MMHB predicted by scalar-tensor theories as a function of $\Upsilon$. One can see that theories with increasingly large values of $\Upsilon$ predict that the MMHB is larger. This is a consequence of the reduced gravity. When $\Upsilon$ is increased, the central temperature and density at fixed mass is reduced since less nuclear burning is needed to provide the pressure gradient to support the star. [Note from Eq. (25) that the luminosity 


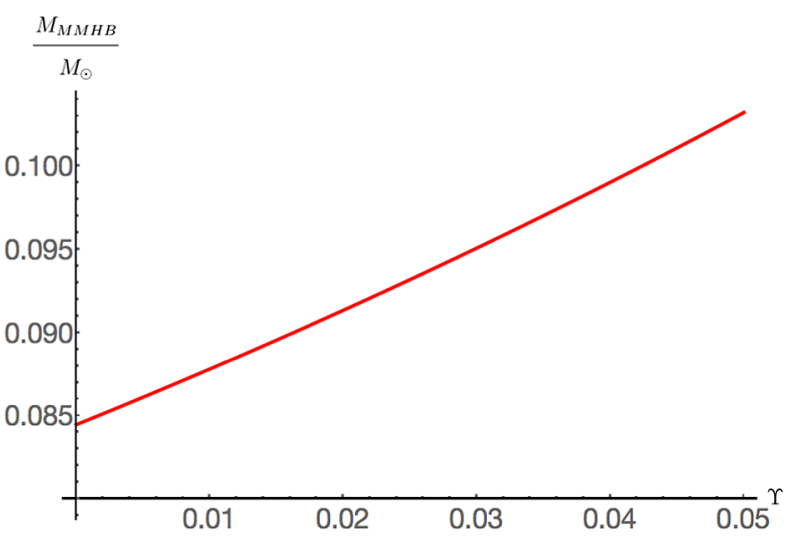

FIG. 1 (color online). The MMHB as a function of $\Upsilon$.

of the photosphere is largely independent of the modifications of gravity and scales in the same manner as the opacity. Most of the deviations from GR are due to changes in the hydrogen burning rate.] One then needs a larger mass to achieve the conditions necessary for hydrogen burning.

It is evident that there are large changes in the MMHB even for small deviations from GR. There have been several low mass red dwarf stars observed in our local neighborhood with masses in the range $0.08 M_{\odot}$ to $0.2 M_{\odot}$ (see Refs. [16-21] and references therein). The lowest mass observed $M$ dwarf is Gl $866 \mathrm{C}$ [18], which has a mass of $0.0930 \pm 0.0008 M_{\odot}$. (There are some stars with measured mass $M \sim 0.08 M_{\odot}$, but the upper limit given by experimental errors exceeds the upper limit for this object.) Using Eq. (26), one finds that this value is achieved when $\Upsilon \approx 0.02665$, and so values larger than this are excluded.

As with any astrophysical test of gravity, one must be mindful of possible degeneracies that can potentially mimic the novel effects, or act to negate them. Here, there are few. Changing $\Upsilon$ is partly degenerate with increasing the opacity, but the MMHB is only weakly sensitive to this: it scales like $\kappa^{0.11}$ and cannot negate the changes coming from the effects of modified gravity on the structure of the star. The MMHB is weakly dependent on the amount of stellar rotation, which is absent in our model. Rotation acts to increase the MMHB $[22,23]$ and so cannot act to negate the effects of modified gravity. Therefore, it is not a caveat to the MMHB predicted in alternative gravity theories.

Finally, one may worry that the empirical mass determination of low mass objects intrinsically assumes GR. This is not the case. The general theories considered here only exhibit deviations from GR inside astrophysical bodies. Outside, Newtonian physics applies. Many of the stars referenced above either exist in eclipsing binary systems or have smaller satellites. In each case, Newtonian mechanics is used to measure their masses and not their intrinsic properties. In other cases, photometry is used to measure the mass using the mass-luminosity relation. Examination of Eq. (25) reveals that this does depend on the theory of gravity; however, the relation used to calculate the photometric mass is an empirical fit to observations using stars of known mass found using the eclipsing binary technique [24]. As such, masses found using this technique are independent of the theory of gravity.

In summary, we have shown here that the most general scalar-tensor theories of gravity predict that the onset of hydrogen burning in low mass stars occurs at higher masses than general relativity predicts. Red dwarf stars with masses larger than $0.08 M_{\odot}$ have been observed, and, indeed, this value is compatible with general relativity. The absence of large degeneracies with non-gravitational physics allows us to confidently rule out the entire region of parameter space where $\Upsilon \gtrsim 0.027$. This has two important implications. First, it rules out the possibility that scalar-tensor theories can alter the properties of main and post-main sequence stars because these are negligible when $\Upsilon \lesssim 0.2$ [13]. Similarly, there are only negligible effects on the rotation curves of galaxies and the lensing of light by dark matter halos.

Second, there are cosmological implications. As remarked above, $\Upsilon$ is given by a specific combination of the parameters appearing in the EFT of dark energy. These parameters completely characterize the cosmology on linear scales and are the focus of upcoming surveys aimed at testing gravity in this regime. Using the novel effect presented here, we can place the independent constraint

$$
\frac{\alpha_{H}^{2}}{\alpha_{H}-\alpha_{T}-\alpha_{B}\left(1+\alpha_{T}\right)} \lesssim 0.0068 \text {. }
$$

This directly restricts the possible deviations from general relativity on cosmological scales, and has the potential to rule out competitors to $\Lambda \mathrm{CDM}$. Indeed, one example of a commonly studied alternative to $\Lambda \mathrm{CDM}$ is the covariant quartic Galileon, which admits a self-accelerating de Sitter solution with $\Upsilon=1 / 3$. The constraint we have placed here is an upper bound on $\Upsilon$. It is interesting to note that stable stellar configurations cannot exist when $\Upsilon \leq-2 / 3$ [14], and so only a narrow window for $\Upsilon$ remains.

What is perhaps most interesting is that the behavior of local objects has the power to elucidate the nature of dark energy. This remains elusive and the constraint we have obtained here clearly has the potential to drastically reduce the space of viable alternative gravity theories.

*jeremy.sakstein@port.ac.uk

[1] A. Joyce, B. Jain, J. Khoury, and M. Trodden, Phys. Rep. 568, 1 (2015).

[2] J. Gleyzes, D. Langlois, F. Piazza, and F. Vernizzi, Phys. Rev. Lett. 114, 211101 (2015).

[3] R. Kase and S. Tsujikawa, Phys. Rev. D 90, 044073 (2014).

[4] A. Barreira, B. Li, C. Baugh, and S. Pascoli, J. Cosmol. Astropart. Phys. 08 (2014) 059. 
[5] T. Kobayashi, Y. Watanabe, and D. Yamauchi, Phys. Rev. D 91, 064013 (2015).

[6] S. S. Kumar, Astrophys. J. 137, 1121 (1963).

[7] A. Burrows and J. Liebert, Rev. Mod. Phys. 65, 301 (1993).

[8] G. Chabrier, I. Baraffe, J. Leconte, J. Gallardo, and T. Barman, AIP Conf. Proc. 1094, 102 (2009).

[9] A. De Felice, T. Kobayashi, and S. Tsujikawa, Phys. Lett. B 706, 123 (2011).

[10] A.-C. Davis, E. A. Lim, J. Sakstein, and D. J. Shaw, Phys. Rev. D 85, 123006 (2012).

[11] V. Vikram, J. Sakstein, C. Davis, and A. Neil, arXiv:1407.6044.

[12] B. Jain, V. Vikram, and J. Sakstein, Astrophys. J. 779, 39 (2013).

[13] K. Koyama and J. Sakstein, Phys. Rev. D 91, 124066 (2015).

[14] R. Saito, D. Yamauchi, S. Mizuno, J. Gleyzes, and D. Langlois, J. Cosmol. Astropart. Phys. 06 (2015) 008.

[15] J. Gleyzes, D. Langlois, F. Piazza, and F. Vernizzi, J. Cosmol. Astropart. Phys. 02 (2015) 018.
[16] D. S. Coppenbarger, T. J. Henry, and D. W. McCarthy, Jr., Astron. J. 107, 1551 (1994).

[17] C. Barbieri, G. De Marchi, A. Nota, G. Corrain, W. Hack, R. Ragazzoni, and D. Macchetto, Astron. Astrophys. 315, 418 (1996).

[18] D. Segransan, X. Delfosse, T. Forveille, J. L. Beuzit, S. Udry, C. Perrier, and M. Mayor, Astron. Astrophys. 364, 665 (2000).

[19] X. Delfosse, T. Forveille, D. Segransan, J.-L. Beuzit, S. Udry et al., Astron. Astrophys. 364, 217 (2000).

[20] F. Martinache, J. P. Lloyd, M. J. Ireland, R. S. Yamada, and P. G. Tuthill, Astrophys. J. 661, 496 (2007).

[21] P. S. Muirhead, J. A. Johnson, K. Apps, J. A. Carter, T. D. Morton, D. C. Fabrycky, J. S. Pineda, M. Bottom, B. Rojas-Ayala, E. Schlawin et al., Astrophys. J. 747, 144 (2012).

[22] E. E. Salpeter, Astrophys. J. 393, 258 (1992).

[23] R. Kippenhahn, Astron. Astrophys. 8, 50 (1970).

[24] T. J. Henry and D. W. McCarthy, Jr., Astron. J. 106, 773 (1993). 